\title{
Discontinuous Legendre Wavelet Galerkin Method for Solving Lane-Emden Type Equation
}

\author{
Xiaoyang Zheng ${ }^{1 *}$, Zhengyuan Wei ${ }^{1}$ and Jiangping $\mathrm{He}^{1}$ \\ ${ }^{1}$ College of Mathematics and Statistics, Chongqing University of Technology, 400054, Chongqing, China \\ Email: zhengxiaoyang@cqut.edu.cn
}

\begin{abstract}
This paper presents a new numerical approach, i.e., discontinuous Legendre wavelet Galerkin (DLWG) technique, to solve the Lane-Emden equations. This scheme incorporates Legendre wavelet into discontinuous Galerkin (DG) method, thus it has the advantages of both wavelet Galerkin (WG) method and DG technique. Specifically, the variational formulation of the equation and numerical fluxes are first derived and clearly calculated, then the Lane-Emden equations are converted into solutions to systems of equations. It is pointed that the proposed approach needs less storage space and execution time than the other methods because of the use of discontinuous elements producing lower dimensional, block-diagonal and sparse mass matrices. Furthermore, numerical experiments demonstrate the efficiency and applicability of this technique.
\end{abstract}

Keywords: Legendre wavelet, Discontinuous Galerkin method, Lane-Emden equation, Discontinuous Legendre wavelet Galerkin method.

\section{Introduction}

Recently, a lot of efforts have been made to solve the Lane-Emden equations with singular initial value problems, which are used to model many phenomena in mathematical physics and astrophysics [1-3]. For example, these equations can describe the equilibrium density distribution in self-gravitating sphere of polytrophic isothermal gas, and characterize the principle of thermionic currents and the theory of stellar structure, etc. In general, the equations mentioned above have a form as follows

$$
u^{\prime \prime}(x)+\frac{\alpha}{x} u^{\prime}(x)+f(x, u)=g(x), \quad 0<x \leq 1, \quad \alpha \geq 0
$$

with the initial conditions

$$
u(0)=A, \quad u^{\prime}(0)=B,
$$

where $A, B$ and $\alpha$ are constants, $f(x, u)$ is a nonlinear continuous function with respect to $x$ and $u$, and $g(x) \in C([0,1])$. Especially, when $\alpha=2, f(x, u)=e^{u}$ and $g(x)=0$, Eq. (1.1) is reduced to the model of the isothermal gas spheres [4-7].

Several crucial techniques, such as Adomian decomposition method, homotopy analysis method, variational approach, perturbation technique and operational matrix methods, have been developed to compute the solution of Eq. (1.1). For example, Wazwaz [5] has given a general study to construct exact and series solution to this equation by employing the Adomian decomposition method. Liao [6] applied the homotopy analysis method to solving Eq. (1.1). Singh et al. [4] introduced modified homotopy analysis method for this equation and obtained analytical approximate solution and Iqbal et al. [7] addressed optimal homotopy method to solve Eq. (1.1). Additionally, He [8] proposed a variational approach for solving this equation. M. Dehghan et al. [9] obtained approximate solution of a differential equation arising in astrophysics using variational iteration method. Parand et al. [10] obtained rational Legendre pseudospectral approach for solving Eq. (1.1). Furthermore, Van Gorder [11] presented perturbation technique to solve this equation. Finally, besides these approaches, we need to introduce 
Legendre wavelet operational matrix methods, which can convert this equation to a system of equations by computing operational matrix of integration or derivative [12-19]. For example, Razzaghi Yousefi [14] derived the Legendre wavelet operational matrix of integration to solve Eq. (1.1). Pandey et al. [17] proposed the Legendre wavelet operational matrix of derivative to solve this equation. Applications of Legendre wavelet techniques for numerical approximations of ODEs can be found in the references [1925]. However, although Legendre wavelet approaches are implemented to solve various ODEs and PDEs, we note that there exist at least two aspects in computational complexity that need to be improved, especially for solving nonlinear ODEs. One is that the dimensions of the operational matrix increase rapidly when the level of resolution is refined. The other is that the operational matrix of derivative should be calculated by another method. The reason is that Legendre wavelet functions are discontinuous at nodes when the order of Legendre wavelet is odd.

In order to cope with the above obstacles, we devise an alternative technique, i.e., the DLWG approach through borrowing the idea of the DG method and utilizing the rich properties of Legendre wavelet bases. More elaborately, the DLWG technique is based on the variational formulation for the solution of Eq. (1.1) and takes advantage of an elementwise discontinuous Legendre wavelet approximation, where the numerical information is only communicated locally via numerical fluxes. Of course, we also evaluate the errors of the calculations for the numerical fluxes. Finally, the DLWG approach containing the above advantages is applied to solving Eq. (1.1). Several numerical experiments for the linear and nonlinear Eqs. (1.1) demonstrate the efficiency and capability of the proposed method.

Compared with the above methods, the essential advantages of the DLWG approach are briefly described as: Sparser mass matrices are derived owing to the rich properties of Legendre wavelet; Lower dimensional mass matrices can be obtained because of the use of the discontinuous element approximation on each subinterval; Legendre wavelet operational matrix of derivative is the same on each subinterval and can avoid the disadvantage of discontinuity of Legendre wavelet at nodes; The singular initial conditions and the discontinuity of Legendre wavelet bases are easier to be coped with at the interface between elements by using the numerical fluxes, which appear from integration by parts. In conclusion, the DLWG technique can obtain satisfactory mass matrices and operational matrix of derivative which can be easily inverted by hand and stored efficiently in computer memory and thus can decrease the computational complexity.

The organization of the paper is as follows. In Section 2, descriptions of Legendre wavelet bases and its rich properties are elaborated. Section 3 derives the weak formulation of Eq. (1.1) by using the DLWG method and the numerical fluxes are suitably selected and clearly computed. Legendre wavelet operational matrix of derivative is calculated. These calculations can convert Eq. (1.1) into a system of equations with respect to the coefficients of Legendre wavelet bases. Then, the system of equations is solved by using Newton's iterative method. In section 4, the method proposed in this article is applied to solving the linear and nonlinear equations of Eqs. (1.1) type. The good results of the numerical experiments show that our method is very effective. Conclusions of the proposed approach in this paper are given at the end in Section 5 .

\section{Preliminaries and Notations}

In this section, we first briefly introduce Legendre wavelet bases and our notations. Secondly, the rich properties and some important results of Legendre wavelet that will be used later are elaborated. In addition, it should be pointed out that Legendre wavelet methods for the numerical solutions of ODEs and PDEs have enjoyed substantial development in recent years [20-25]. Therefore, the Legendre wavelet bases can offer an efficient tool for the numerical solution of Eq. (1.1). 


\section{$2.1 \quad$ Legendre Wavelet}

For decomposition level $n=0,1, \cdots$ and translation $l=0,1,2, \cdots, 2^{n}-1$, we define the subinterval, i.e., element $I_{n l}=\left[2^{-n} l, 2^{-n}(l+1)\right)$. For $p=1,2, \cdots$, define $V_{p, n}$ as a subspace of piecewise polynomial functions satisfying

$V_{p, n}=\left\{f:\left.f\right|_{I_{n l}}\right.$ is a polynomial of degree strictly less than $p$; and $f$ vanishes elsewhere $\}$, which constitutes a linear space.

We now start to review Legendre polynomials and Legendre wavelet bases. Let $L_{k}(x)$ denote Legendre polynomial of degree $k$, which is defined as follows:

$$
\begin{gathered}
L_{0}(x)=1, \quad L_{1}(x)=x, \\
L_{k+2}(x)=\frac{2 k+3}{k+2} x L_{k+1}(x)-\frac{k+1}{k+2} L_{k}(x) .
\end{gathered}
$$

Then, at the level of resolution $n=0$, let $\varphi_{k}(x)$ denote Legendre scale functions defined as

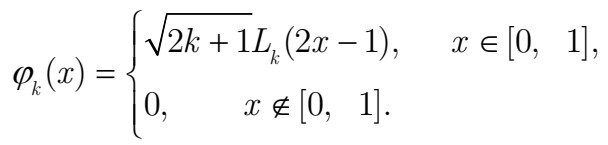

The whole set $\left\{\varphi_{k}\right\}_{k=0}^{p-1}$ forms an orthonormal basis for $V_{p, 0}$. Generally, the subspace $V_{p, n}$ is spanned by $2^{n} p$ functions which are obtained from $\varphi_{0}, \cdots, \varphi_{k-1}$ by dilation and translation, i.e.,

$$
V_{p, n}:=V_{p, n l}=\operatorname{span}\left\{\varphi_{k, n l}(x)=2^{n / 2} \varphi_{k}\left(2^{n} x-l\right), 0 \leq k \leq p-1,0 \leq l \leq 2^{n}-1\right\}
$$

which forms an orthonormal basis for $L_{2}([0,1])$ and

$$
V_{p, 0} \subset V_{p, 1} \subset \cdots \subset V_{p, n} \subset \cdots .
$$

In order to intuitively understand Legendre scale functions at different level $n$ of resolution, let $p=3$, the coarsest scale $n=0$ and the finest scale $n=2$, then Fig.1 plots the coarsest scale functions $\varphi_{k}(x)$, and Fig.2 illustrates the finest scale functions $\varphi_{k, 2 l}(x)$, respectively.

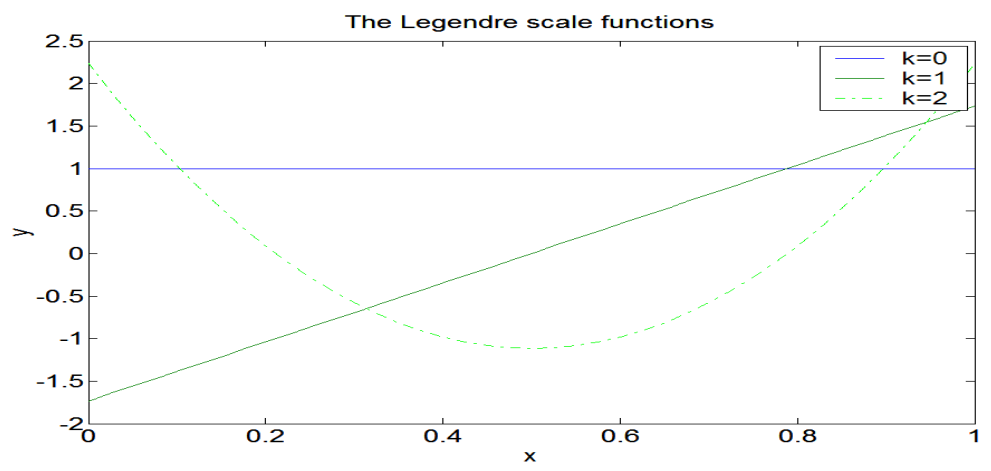

Fig.1. Legendre scale functions with $n=0$ and $p=3$. 


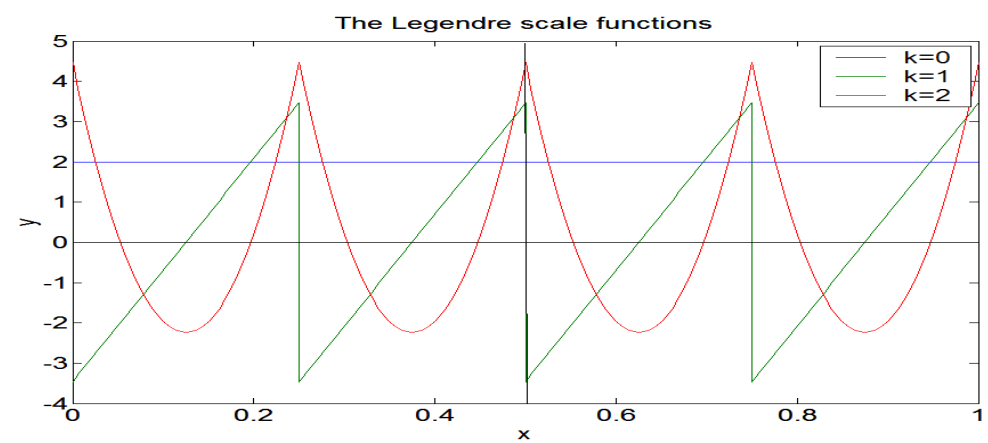

Fig.2. Legendre scale bases with $n=2$ and $p=3$.

\subsection{Some Properties of the Legendre Wavelet}

This subsection briefly describes the rich properties of Legendre wavelet bases. We shall know that these results play an important role in solving Eq. (1.1) by using DLWG approach.

Orthogonality: The set of Legendre wavelet bases in subspace $V_{p, n}$ is an orthogonal set as

$$
\int_{2^{-n} l}^{2^{-n}(l+1)} \varphi_{k, n l}(x) \varphi_{k^{\prime}, n l^{\prime}}(x) \mathrm{d} x=\delta_{k k^{\prime}} \delta_{l^{\prime}} .
$$

which is very easy to prove by variable substitution because Legendre polynomials defined on interval $[-1,1]$ are orthogonal.

Approximation: A function $u \in L_{2}([0,1])$ in $V_{p, n}$ is approximated by Legendre wavelet bases as follows

$$
\mathbf{P}_{n} u(x)=\sum_{l=0}^{2^{n}-1} \sum_{k=0}^{p-1} c_{k, n} \varphi_{k, n l}(x),
$$

where $P_{n}$ is the projection at the finest scale $n$, and the scale coefficients $c_{k, n l}$ are calculated via

$$
c_{k, n l}=\int_{I_{n l}} u(x) \varphi_{k, n l}(x) \mathrm{d} x
$$

where the coefficients $c_{k, n l}$ and scale bases $\varphi_{k, n l}(x)$ are denoted by vectors as

$$
\begin{aligned}
C & =\left[c_{0, n 0}, \cdots, c_{p-1, n 0}, c_{0, n 1}, \cdots, c_{p-1, n 1}, \cdots, c_{0, n, 2^{n}-1}, \cdots, c_{p-1, n, 2^{n}-1}\right]^{T}, \\
\Phi_{p, n} & =\left[\varphi_{0, n 0}, \cdots, \varphi_{p-1, n 0}, \varphi_{0, n 1}, \cdots, \varphi_{p-1, n 1}, \cdots, \varphi_{0, n, 2^{n}-1}, \cdots, \varphi_{p-1, n, 2^{n}-1}\right]^{T} .
\end{aligned}
$$

Using (2.4), numerical computation can be done by Legendre wavelet technique.

Values of Legendre wavelet: The evaluations of Legendre wavelet at nodes $x=2^{-n} l$ and $x=2^{-n}(l+1)$ are

$$
\varphi_{k, n l}\left(2^{-n} l\right)=(-1)^{k} 2^{n / 2} \sqrt{2 k+1}, \quad \varphi_{k, n l}\left(2^{-n}(l+1)\right)=2^{n / 2} \sqrt{2 k+1},
$$

which can be obtained by using the following properties of Legendre polynomials

$$
L_{k}(1)=1, \quad L_{k}(-1)=(-1)^{k} .
$$

These results are applied to computing the fluxes.

Relation between the function and the derivative: Using a relation for Legendre polynomials

$$
(2 k+1) L_{k}(x)=L_{k+1}^{\prime}(x)-L_{k-1}^{\prime}(x),
$$

we can obtain an explicit representation for the first derivative such that

$$
\frac{\varphi_{j}^{\prime}(x)}{2 \sqrt{2 j+1}}=\sqrt{2 j-1} \varphi_{j-1}(x)+\sqrt{2 j-5} \varphi_{j-3}(x)+\cdots+\left\{\begin{array}{lll}
\varphi_{0}(x), & j & \text { odd }, \\
\sqrt{3} \varphi_{1}(x), & j & \text { even. } .
\end{array}\right.
$$


We shall utilize the above nice properties of Legendre wavelet bases to compute each term of the following weak formulation of Eq. (1.1).

\section{Computations of the Weak Formulation of the Equation}

In this section, we first derive the variational formulation of Eq. (1.1). Secondly, we obtain the operational matrix of derivative. Thirdly, we analyze how to compute the product operator of the Legendre wavelet functions and integer power operator of the function $u$. Furthermore, these computational results are implemented to convert the weak formulation of Eq. (1.1) into a system of equations on each element $I_{n l}$.

\subsection{Variational Formulation of the Equation}

We now first define some notations to clearly describe the DLWG approach. We utilize $u_{l}^{+}$and $u_{l}^{-}$to denote the values of $u$ at $x_{l}=2^{-n} l$ and $l=1,2, \cdots, 2^{n}-1$ from right and left, respectively,

$$
u_{l}^{+}=\lim _{\varepsilon \rightarrow 0+} u\left(x_{l}+\varepsilon\right), \quad u_{l}^{-}=\lim _{\varepsilon \rightarrow 0+} u\left(x_{l}-\varepsilon\right) .
$$

We also let the usual notations $\{u\}=\left(u^{+}+u^{-}\right) / 2$ and $[[u]]=u^{+}-u^{-}$represent the mean and the jump of the function $u$ at each element $I_{n l}$ boundary point, respectively. Secondly, for each subinterval, i.e., element $I_{n l}$, we seek an approximation $u_{h}$ to $u$ such that $u_{h}$ is the $p$ dimensional subspace $V_{p, n l}$ for a certain $l$ and is represented as the (2.4), where $h$ denotes the size of each element. In order to determine the approximate solution $u_{h}$, we use a weak formulation, i.e., we multiply Eq. (1.1) by arbitrary smooth functions $v$, and integrate over a certain element $I_{n l}$ and have

$$
\int_{I_{n l}} u^{\prime \prime} v \mathrm{~d} x+\alpha \int_{I_{n l}} \frac{u^{\prime}}{x} v \mathrm{~d} x+\int_{I_{n l}} f(x, u) v \mathrm{~d} x=\int_{I_{n l}} g(x) v \mathrm{~d} x .
$$

Then, by using a simple formal integration by parts, we obtain

$$
\begin{gathered}
-\int_{I_{n l}} u^{\prime} v^{\prime} \mathrm{d} x-\alpha \int_{I_{n l}} u \frac{v^{\prime} x-v}{x^{2}} \mathrm{~d} x+\left(u_{x_{l+1}}^{\prime}+\alpha u_{x_{l+1}} / x\right) v_{x_{l+1}}^{-}-\left(u_{x_{l}}^{\prime}+\alpha u_{x_{l}} / x\right) v_{x_{l}}^{+} \\
=\int_{I_{n l}} g(x) v \mathrm{~d} x-\int_{I_{n l}} f(x, u) v \mathrm{~d} x .
\end{gathered}
$$

Next, we replace the smooth functions $v$ by test functions $\varphi_{k, n l}$ which are in the discontinuous finite element space $V_{p, n}$, and the exact solution $u$ by the approximate solution $u_{h}$. Then, we obtain the weak formulation of Eq.(1.1) by the DLWG technique discretization satisfying

$$
\begin{gathered}
-\int_{I_{n l}} u_{h}^{\prime} \varphi_{k, n l}^{\prime} \mathrm{d} x-\alpha \int_{I_{n l}} u_{h} \frac{\varphi_{k, n l}^{\prime} x-\varphi_{k, n l}}{x^{2}} \mathrm{~d} x+\left[\left(\overline{u_{h}^{\prime}}\right)_{x_{l+1}}+\alpha\left(\overline{u_{h}}\right)_{x_{l+1}} / x\right]\left(\varphi_{k, n l}^{-}\right)_{x_{l+1}}-\left[\left(\overline{u_{h}^{\prime}}\right)_{x_{l}}+\alpha\left(\overline{u_{h}}\right)_{x_{l}} / x\right]\left(\varphi_{k, n l}^{+}\right)_{x_{l}} \\
=\int_{I_{n l}} g(x) \varphi_{k, n l} \mathrm{~d} x-\int_{I_{n l}} f\left(x, u_{h}\right) \varphi_{k, n l} \mathrm{~d} x
\end{gathered}
$$

where $\overline{u_{h}^{\prime}}$ and $\overline{u_{h}}$ are the fluxes. Since Legendre wavelet functions with odd order are discontinuous at the end points of each subinterval $I_{n l}$, we must also replace the $u_{h}^{\prime}$ and $u_{h}$ by the numerical fluxes $\overline{u_{h}^{\prime}}$ and $\overline{u_{h}}$, respectively, which depend on the two values of $u_{h}$ or $u_{h}^{\prime}$ at the end point, and are suitably chosen as

$$
\overline{u_{h}^{\prime}}=\beta_{0}\left[\left[u_{h}\right]\right]+\left\{u_{h}^{\prime}\right\}
$$

where $\beta_{0}$ is a parameter, and 


$$
\overline{u_{h}}=\left\{u_{h}\right\}-\gamma\left[\left[u_{h}\right]\right],
$$

which is the local Lax-Friedrichs flux and $\gamma=\max _{u}\left|u^{\prime}\right|$, refer to [25] for more details.

In order to effectively evaluate each term in (3.3) and convert it into a system of equations, we need to compute the operational matrix of derivative, the product operation of Legendre wavelet vector bases and the integer power operator of a function.

\subsection{Computing the Operational Matrix of Derivative}

In this subsection we derive Legendre wavelet operational matrix of derivative on each element $I_{n l}$. Let $D$ denote the derivative operator and consider $D_{p, n}: V_{p, n} \rightarrow V_{p, n}$ (the projection $P_{p, n} D P_{p, n}$ of $D$ on $\left.V_{p, n}\right)$. Then let us consider solution $u$ and $P_{p, n} u, D_{p, n} u \in V_{p, n}$ with expansions by using Legendre wavelet functions, respectively,

$$
\begin{gathered}
\left(P_{p, n} u\right)(x)=\sum_{m=0}^{2^{n}-1} \sum_{j=0}^{p-1} s_{j, n m} \varphi_{j, n m}(x), \\
\left(D_{p, n} u\right)(x)=\sum_{l=0}^{2^{n}-1} \sum_{i=0}^{p-1} \overline{s_{i, n l}} \varphi_{i, n l}(x) .
\end{gathered}
$$

Our goal is to find the $p \times p$ transition matrices $r_{n l m}$ for $l, m=0,1, \cdots, 2^{n}-1$, which satisfy

$$
\overline{s_{i, n l}}=\sum_{m=0}^{2^{n}-1} \sum_{j=0}^{p-1}\left[r_{n l m}\right]_{i j} s_{j, n m} .
$$

Then the coefficient $\left[r_{n l m}\right]_{i j}$ would necessarily be given by

$$
\left[r_{n l m}\right]_{i j}=\int_{2^{-n} l}^{2^{-n}(l+1)} \varphi_{i, n l}(x) D \varphi_{j, n m}(x) \mathrm{d} x=2^{n}\left[r_{l-m}\right]_{i j},
$$

where

$$
\left[r_{l^{\prime}}\right]_{i j}=\int_{0}^{1} \varphi_{i}(x) D \varphi_{j}\left(x+l^{\prime}\right) \mathrm{d} x
$$

is the representation of $D$ on the coarsest scale $V_{p, 0}$. Also, because we compute the transition matrices on each element $I_{n l}$, only the same element is involved, that is $l^{\prime}=0$. Consequently, the matrix in (3.10) is replaced by

$$
\left[r_{0}\right]_{i j}=\int_{0}^{1} \varphi_{i}(x) \frac{d}{d x} \varphi_{j}(x) \mathrm{d} x,
$$

which again is a formal expression at this point, where $i, j=0,1, \cdots, p-1$ and $r_{0}$ is a $p \times p$ matrix. Additionally, the representation of transition matrix can be found on each element $I_{n l}$ by rescaling

$$
r_{n, 0}=2^{n} r_{0} .
$$

We now describe how to provide explicit calculation for each element of the matrix in (3.11) for Legendre wavelet bases. Substituting (2.9) into (3.11), we find that the $(i+1, j+1)$ th element of the matrix $\left(r_{0}\right)_{i+1, j+1}=\left[r_{0}\right]_{i j}$ satisfies

$$
\left(r_{0}\right)_{i+1, j+1}=2 \Gamma_{i j} v_{i j},
$$

where $\Gamma_{i j}=\sqrt{2 i+1} \sqrt{2 j+1}$ and

$$
v_{i j}= \begin{cases}1, & j-i=1,3,5, \cdots, \\ 0, & \text { otherwise. }\end{cases}
$$

For example, let $p=3$, we can obtain 


$$
r_{0}=2\left(\begin{array}{ccc}
0 & \sqrt{3} & 0 \\
0 & 0 & \sqrt{15} \\
0 & 0 & 0
\end{array}\right) .
$$

Furthermore, what it attracts our attentions most is that the matrix is the same at each element $I_{n l}$ for every $l$. This result is easy to be proved by using variable substitution. In addition, if the decomposition level $n$ is large enough, then the lower dimensional operational matrix of derivative can simplify the storage and computational complexity when solving a system of equations. Thus, this technique provides a new method for solving differential equations.

\subsection{Computation of Integer Power Operator}

This subsection calculates the product operation of Legendre wavelet vector bases and the integer power operator of a function $u$ approximated by Legendre wavelet on the element $I_{n l}$, respectively.

The product operation of Legendre wavelet vector bases is approximated as

$$
Q_{0} \Phi_{p, n l}(x) \Phi_{p, n l}(x)^{T} \approx \Phi_{p, n l}(x)^{T} Q,
$$

where $Q_{0}=\left(g_{0, n l}, g_{1, n l}, \cdots, g_{p-1, n l}\right), \quad \Phi_{p, n}(x)=\left(\varphi_{0, n l}, \varphi_{1, n l}, \cdots, \varphi_{p-1, n l}\right)^{T}$ and $Q$ is a $p \times p$ dimensional matrix. For $p=3, n=1$ and $l=0$, Avudainay agarn et al. [22] gave this product operation such that

$$
Q_{0} \Phi_{3,1}(x) \Phi_{3,1}(x)^{T} \approx \Phi_{3,1}(x)^{T}\left(\begin{array}{ccc}
\sqrt{2} g_{0,10} & \sqrt{2} g_{1,10} & \sqrt{2} g_{2,10} \\
\sqrt{2} g_{1,10} & \sqrt{2} g_{0,10}+\frac{4}{\sqrt{10}} g_{2,10} & \frac{4}{\sqrt{10}} g_{1,10} \\
\sqrt{2} g_{2,10} & \frac{4}{\sqrt{10}} g_{1,10} & \sqrt{2} g_{0,10}+\frac{20}{7 \sqrt{10}} g_{2,10}
\end{array}\right),
$$

where the element of the matrix of the product operation is composed of linear combinations of elements of the vector $Q_{0}$. In this paper, we address the general computational procedure for this product operation by using the DLWG method, which is similar to that of the literature [24]. For any fixed element $I_{n l}$, we let

$$
Q_{p \times p}=\left(\begin{array}{ccc}
a_{00} & \cdots & a_{0, p-1} \\
\cdots & \cdots & \cdots \\
a_{p-1,0} & \cdots & a_{p-1, p-1}
\end{array}\right),
$$

where

$$
a_{i j}=\int_{2^{-n} l}^{2^{-n}(l+1)} \varphi_{i, n l}(x)\left(\sum_{k=0}^{p-1} g_{k, n l} \varphi_{k, n l}(x) \varphi_{j, n l}(x)\right) d x,
$$

$i, j=0,1, \cdots, p-1$ and the matrix $Q_{p \times p}$ is symmetric. By simple variable substitute, it is easy to prove this matrix is the same on every element $I_{n l}$.

We now address the integer power of a function $u(x)$, which may be approximated as

$$
[u(x)]^{\tau}=\left[C^{T} \Phi_{p, n l}(x)\right]^{\tau} \approx C_{\tau} \Phi_{p, n l}(x),
$$

where $C_{\tau}$ is a vector, whose elements are nonlinear combinations of the elements of the vector $C_{l}$, where $C_{l}$ denotes the part of translation $l$ in (2.4). Similar to the computation of the product operation mentioned above, the $C_{\tau}$ can be calculated. 
Finally, we briefly discuss an algorithm for evaluating the nonlinear function $f(x, u)$ with respect to $x$ and $u$. Assume $f(x, u)$ to be analytic, such as $f(x, u)=u^{\tau}$ or $f(x, u)=e^{u}$, we substitute Taylor series with respect to $u$ to get

$$
f(x, u)=\sum_{k=0}^{N} \frac{f^{(k)}\left(u_{0}\right)}{k !}\left(u-u_{0}\right)^{k}+E_{n, N}(f, u),
$$

where $u_{0}$ is the initial condition and $E_{n, N}(f, u)$ is the error term that is controlled by choosing decomposition level $n$ or inter power $N$ sufficiently large. Then, the calculations of the power term in (3.19) are finished by using the inter power presentations approach in (3.18). This technique is implemented to compute function $f(x, u)$ that has rapidly converging Taylor series expansions. Consequently, the nonlinear term $f\left(x, u_{h}\right)$ of the variational formulation in (3.3) can be effectively calculated by using this evaluating technique.

These effective calculations derived above are very suitable for converting the discontinuous varivational formulation (3.3) into a system of equations for each translation $l$.

\subsection{Converting Lane-Emden Equation into a System of Equations}

In this subsection, we present the main characteristic of the DLWG technique for solving Eq.(1.1), which is that it reduces these problems to those of solving a system of equations on each element $I_{n l}$ for all $k=0,1, \cdots, p-1$, thus greatly simplifying the problems. We now return to specifically compute each term of the variational formulation of Eq. (1.1) in (3.3).

For the first term in (3.3) and all test functions $\varphi_{k, n l}, k=0,1, \cdots, p-1$, we use the operational matrix of derivative in (3.12) to have

$$
-\int_{I_{n l}} u_{h}^{\prime} \varphi_{k, n l}^{\prime} d x=-2^{n} r_{0}^{T} r_{0} C_{l}
$$

For the second and third terms in (3.3), the functions $1 / x$ and $1 / x^{2}$ can be approximated as

$$
1 / x=E_{01}^{T} \Phi_{p, n l}(x), \quad 1 / x^{2}=E_{02}^{T} \Phi_{p, n l}(x),
$$

where $E_{01}, E_{02}$ are the approximate coefficients, respectively. These coefficients can be computed by (2.5) through substituting $1 / x, 1 / x^{2}$ for $u$, respectively. Then, with (3.12) and (3.18), we obtain

$$
-\alpha \int_{I_{n l}} u_{h} \frac{\varphi_{k, n l}^{\prime} x-\varphi_{k, n l}}{x^{2}} d x=-\alpha 2^{n}\left(r_{0}^{T} E_{1}-E_{2}\right) C_{l},
$$

where the elements of $E_{1}, E_{2}$ are the linear combinations of those of the vector $E_{01}, E_{02}$ in terms of (3.21), respectively.

For the two terms on the right of (3.3), the function $g(x)$ is represented as

$$
g(x)=G^{T} \Phi_{p, n l}(x),
$$

where $G$ is the approximate coefficient, which is similarly calculated by (2.5). Using the results in (3.18) and (3.19) derived in subsection 3.3, the nonlinear function $f\left(x, u_{h}\right)$ can be approximated as

$$
f\left(x, u_{h}\right)=F_{C} \Phi_{p, n l}(x),
$$

where the elements of vector $F_{C}$ are the nonlinear combinations of those of the vector $C_{l}$. With (3.22) and (3.23), we can obtain

$$
\int_{I_{n l}} g(x) \varphi_{k, n l} d x-\int_{I_{n l}} f\left(x, u_{h}\right) \varphi_{k, n l} d x=G-F_{C_{l}}
$$


We now discuss how to compute the fluxes in (3.3). In order to effectively calculate the fluxes $\overline{u_{h}^{\prime}}$ and $\overline{u_{h}}$, we should adequately evaluate the $\left[\left[u_{h}\right]\right],\left\{u_{h}\right\}$ and $\left\{u_{h}^{\prime}\right\}$. Thus, we must first calculate the values of the $u^{+}$and $u^{-}$with needed accuracy at the interface $x=x_{l}$. For $l=1,2, \cdots, 2^{n}-1$, using the property in (2.8), we obtain

$$
\begin{aligned}
u^{+}\left(x_{l}\right) & =u^{+}\left(x_{l+1}\right)=C_{l}^{T} \Phi_{p, n l}\left(x_{l+1}=(l+1) / 2^{n}\right) \\
& =2^{n / 2} C^{T}\left[1,-\sqrt{3}, \cdots,(-1)^{k} \sqrt{2 k+1}, \cdots,(-1)^{p-1} \sqrt{2 p-1}\right]^{T} \\
& =2^{n / 2} C_{l}^{T} \Phi_{(-1)^{k} c}
\end{aligned}
$$

where $C_{l}^{T}$ denotes the coefficients of the $C$ on the $l$ element and let

$$
\Phi_{(-1)^{k} c}=\left[1,-\sqrt{3}, \cdots,(-1)^{k} \sqrt{2 k+1}, \cdots,(-1)^{p-1} \sqrt{2 p-1}\right]^{T} .
$$

Similarly,

$$
u^{-}\left(x_{l}\right)=u^{-}\left(x_{l+1}\right)=2^{n / 2} C_{l}^{T}[1, \sqrt{3}, \cdots, \sqrt{2 k+1}, \cdots, \sqrt{2 p-1}]^{T}=2^{n / 2} C_{l}^{T} \Phi_{c} .
$$

Using (3.25) and (3.26), we have

$$
\left.\frac{\partial u_{h}^{+}}{\partial x}\right|_{x_{l}}=\left.\frac{\partial u_{h}^{+}}{\partial x}\right|_{x_{l+1}}=2^{3 n / 2} C_{l}^{T} r_{0}^{T} \Phi_{(-1)^{k} c},\left.\quad \frac{\partial u_{h}^{-}}{\partial x}\right|_{x_{l}}=\left.\frac{\partial u_{h}^{-}}{\partial x}\right|_{x_{l+1}}=2^{3 n / 2} C_{l}^{T} r_{0}^{T} \Phi_{c} .
$$

In addition, for $l=0$, the initial conditions are substituted into these computations. Using the above results, we have

$$
\begin{gathered}
{\left[\left[u_{h}\right]\right]_{x_{l}}=\left(u_{h}\right)_{x_{l}}^{+}-\left(u_{h}\right)_{x_{l}}^{-}=2^{n / 2} C_{l}^{T}\left(\Phi_{(-1)^{k} c}-\Phi_{c}\right),} \\
\left\{u_{h}\right\}_{x_{l}}=\left[\left(u_{h}\right)_{x_{l}}^{+}+\left(u_{h}\right)_{x_{l}}^{-}\right] / 2=2^{n / 2} C_{l}^{T}\left(\Phi_{(-1)^{k} c}+\Phi_{c}\right) / 2, \\
\left\{u_{h}^{\prime}\right\}_{x_{l}}=\left[\left(u_{h}^{\prime}\right)_{x_{l}}^{+}+\left(u_{h}^{\prime}\right)_{x_{l}}^{-}\right] / 2=2^{3 n / 2} C_{l}^{T} r_{0}^{T}\left(\Phi_{(-1)^{k} c}+\Phi_{c}\right) / 2 .
\end{gathered}
$$

Up to present, we can obtain the computations of the fluxes in (3.4) and (3.5) such that

$$
\begin{gathered}
\overline{u_{h}^{\prime}}=\beta_{0}\left[\left[u_{h}\right]\right]+\left\{u_{h}^{\prime}\right\}=\beta_{0} 2^{n / 2} C_{l}^{T}\left(\Phi_{(-1)^{k} c}-\Phi_{c}\right)+2^{3 n / 2-1} C_{l}^{T} r_{0}^{T}\left(\Phi_{(-1)^{k} c}+\Phi_{c}\right)=\beta_{0} C_{l}^{T} \Phi_{c, k}^{\prime}, \\
\overline{u_{h}}=\left\{u_{h}\right\}-\gamma\left[\left[u_{h}\right]\right]=2^{n / 2-1} C_{l}^{T}\left(\Phi_{(-1)^{k} c}+\Phi_{c}\right)-\gamma 2^{n / 2} C_{l}^{T}\left(\Phi_{(-1)^{k} c}-\Phi_{c}\right)=\gamma C_{l}^{T} \Phi_{c, k},
\end{gathered}
$$

and let $\beta_{0} C_{l}^{T} \Phi_{c, k}^{\prime}=\beta_{0} 2^{n / 2} C_{l}^{T}\left(\Phi_{(-1)^{k} c}-\Phi_{c}\right)+2^{3 n / 2-1} C_{l}^{T} r_{0}^{T}\left(\Phi_{(-1)^{k} c}+\Phi_{c}\right)$, we have

$$
\begin{aligned}
& {\left.\left[\left(\overline{u_{h}^{\prime}}\right)_{x_{l+1}}+\alpha\left(\overline{u_{h}}\right)_{x_{l+1}} / x_{l+1}\right]\left(\varphi_{k, n l}^{-}\right)_{x_{l+1}}-\left[\left(\overline{u_{h}^{\prime}}\right)_{x_{l}}+\alpha \overline{\left(u_{h}\right.}\right)_{x_{l}} / x_{l}\right]\left(\varphi_{k, n l}^{+}\right)_{x_{l}} } \\
= & \beta_{0} C_{l}^{T} \Phi_{c, k}^{\prime}\left(\Phi_{c}-\Phi_{(-1)^{k} c}\right)+\left[\alpha 2^{n} \gamma C_{l}^{T} \Phi_{c, k} /(l+1)\right] \Phi_{c}-\left[\alpha 2^{n} \gamma C_{l}^{T} \Phi_{c, k} / l\right] \Phi_{(-1)^{k} c} \\
= & \alpha C_{l}^{T}\left(\Phi_{c}-\Phi_{(-1)^{k} c}\right) .
\end{aligned}
$$

For all $k=0,1, \cdots, p-1$ and each element $I_{n l}$, with (3.20), (3.21), (3.22) and (3.23), we can convert the discontinuous variational formulation (3.3) into a system of equations satisfying

$$
-2^{n} r_{0}^{T} r_{0} C_{l}-2^{n} \alpha\left(r_{0}^{T} E_{1}-E_{2}\right) C_{l}+\alpha C_{l, \beta_{0} \gamma}\left(\Phi_{c}-\Phi_{(-1)^{k} c}\right)=G-F_{C_{l}}
$$

This system of equations is solved for coefficients $C_{l}$ by using Newton's iterative method and then the numerical solution is obtained on the element $I_{n l}$. For each $k=0,1, \cdots, p-1$, we use the above technique to obtain the numerical solution on every element. Consequently, the numerical solution of Eq. (1.1) can be derived by merging all the solutions on each element.

In comparison with the other Legendre wavelet methods mentioned in Section 1, we do have more equations to solve. However, the reason why our method is valid is that we do not try to solve a large $2^{n} p$ system; rather, we solve $2^{n}$ small $p$ systems with higher efficiency. If the level $n$ of resolution 
is large enough, the lower dimensional operational matrix and the integer power operator derived in Section 3 would remarkably reduce the storage and time cost to solve the full systems.

\subsection{Error Estimates of the Fluxes}

Since we compute the values of the fluxes $\overline{u_{h}^{\prime}}$ and $\overline{u_{h}}$ containing the calculations of the $\left[\left[u_{h}\right]\right]$ and $\left\{\left(u_{h}\right)_{x}\right\}$ at the interface $x=x_{l+1}$ by using the combinations of the values of the scale functions, the computational error incurred should be evaluated. Owing to the nice properties of Legendre wavelet bases, we can obtain better estimate of the numerical fluxes.

Theorem (Fluxes approximation). Let $u \in W^{k}(I)$ and $\mathrm{P} u$ be the projection solution by Legendre wavelet on the decomposition level $n$. Then we can obtain the estimates of the fluxes satisfying

$$
\left|\overline{u_{h}^{\prime}}\right| \leq C 2^{-n(k-1)} \text { and }\left|\overline{u_{h}}\right| \leq C 2^{-n k} \text {, }
$$

where $C$ is a constant.

Proof. We expand $u$ by Legendre wavelet bases as

$$
\mathrm{P} u=\sum_{l=0}^{2^{n}-1} \sum_{k=0}^{p-1} s_{k, n l}(t) \varphi_{k, n l}(x)
$$

and let $x=x_{l+1}$. Alpert et al. [20] derives the estimates for the errors of the forms

$$
\begin{gathered}
u_{l+1}^{-}\left(x=x_{l+1}\right)=2^{n / 2} \sum_{k=0}^{p-1} s_{k, n l} \varphi_{k}(1)+\frac{k !}{(2 k) !} u_{l+1}^{(k)} 2^{-n k}+O\left(2^{-n(k+1)}\right), \\
u_{l+1}^{+}\left(x=x_{l+1}\right)=2^{n / 2} \sum_{k=0}^{p-1} s_{k, n(l+1)} \varphi_{k}(0)+(-1)^{k} \frac{k !}{(2 k) !} u_{l+1}^{(k)} 2^{-n k}+O\left(2^{-n(k+1)}\right) .
\end{gathered}
$$

Thus, we have the estimate of $\mid\left[\left[u_{h}\right]|| \leq C_{0} 2^{-n k}\right.$, which finishes the estimate of $\overline{u_{h}}$. Similarly, we can obtain the other estimate, for details, we refer to [20].

\section{$4 \quad$ Numerical Experiments}

In this section, in order to verify our approach, a few numerical experiments including the linear and nonlinear Lane-Emden type equation given in [5] are implemented by the DLWG approach. The exact solutions of these examples are known so that we can calculate and compare the exact errors and illustrate the efficiency of this method.

Experiment 1: In Eq. (1.1), let $\alpha=2, f(x, u)=u$ and $g(x)=x^{3}+x^{2}+12 x+6$, we have

$$
u^{\prime \prime}+\frac{2}{x} u^{\prime}+u=x^{3}+x^{2}+12 x+6,0 \leq x \leq 1,
$$

with initial conditions $u(0)=0$ and $u^{\prime}(0)=0$, which has exact solution $u(x)=x^{2}+x^{3}$. We solve this problem by using the DLWG method in the different order $p$ of Legendre wavelet and the different scale level $n$ of resolution, and then only present the approximate coefficients in scale space $V_{4,1}$, i.e., $p=4$ and $n=1$, and $c_{k, 1 l}$ satisfies

$$
C_{4,1}=\left[\begin{array}{lllll}
0.0810 & 0.0730 & 0.0230 & 0.0018 & 0 \\
0.7439 & 0.3192 & 0.0428 & 0.0018 & 0
\end{array}\right] .
$$

Futhermore, the mean square error (MSE) between the exact solution and the numerical solution has been calculated and tabulated (see Table 1) at 100 points per element. From the results in Table 1, we know that the required accuracy of the numerical solution of Eq.(4.1) can obtained by selecting the two parameters $p$ and $n$. 
Table 1. Error by using the DLWG method for Example 1

\begin{tabular}{cll}
\hline$p$ & $n$ & \multicolumn{1}{c}{ MSE } \\
\hline \multirow{2}{*}{2} & 1 & $2.53 \times 10^{-2}$ \\
& 2 & $7.8425 \times 10^{-4}$ \\
3 & 1 & $8.2564 \times 10^{-16}$ \\
& 2 & $9.5424 \times 10^{-16}$ \\
4 & 1 & $7.2561 \times 10^{-16}$ \\
& 2 & $5.6484 \times 10^{-16}$ \\
\hline
\end{tabular}

Experiment 2: We consider Lane-Emden equation

$$
u^{\prime \prime}+\frac{2}{x} u^{\prime}-2\left(2 x^{2}+3\right) u=0, \quad 0 \leq x \leq 1,
$$

with the initial conditions $u(0)=1, u^{\prime}(0)=0$. It is known that the exact solution of this equation is $y(x)=e^{x^{2}}$, which can be used for comparing exact error. Table 2 demonstrates the comparison of the exact and numerical solution at 100 points per element for the different order $p$ and decomposition level $n$.

Table 2. Error by using the DLWG method for Example 2

\begin{tabular}{cll}
\hline$p$ & $n$ & \multicolumn{1}{c}{ MSE } \\
\hline \multirow{2}{*}{3} & 1 & $8.07 \times 10^{-2}$ \\
& 2 & $6.4767 \times 10^{-4}$ \\
4 & 1 & $3.3876 \times 10^{-5}$ \\
& 3 & $1.0753 \times 10^{-9}$ \\
5 & 1 & $8.1854 \times 10^{-7}$ \\
& 2 & $2.5549 \times 10^{-11}$ \\
\hline
\end{tabular}

Additionally, we only describe the approximate coefficients $c_{k, 1 l}$ in the scale $V_{4,1 l}$ as

$$
C_{4,1}=\left[\begin{array}{lllll}
0.7707 & 0.0565 & 0.0164 & 0.0010 & 0.0001 \\
1.2975 & 0.2840 & 0.0515 & 0.0062 & 0.0007
\end{array}\right]
$$

Fig. 3 illustrates the exact and numerical solution of Eq. (4.2) in subspace $V_{4,1 l}$. In Fig.3, the '+' and 'o' denote the exact solution and the numerical solution, respectively.

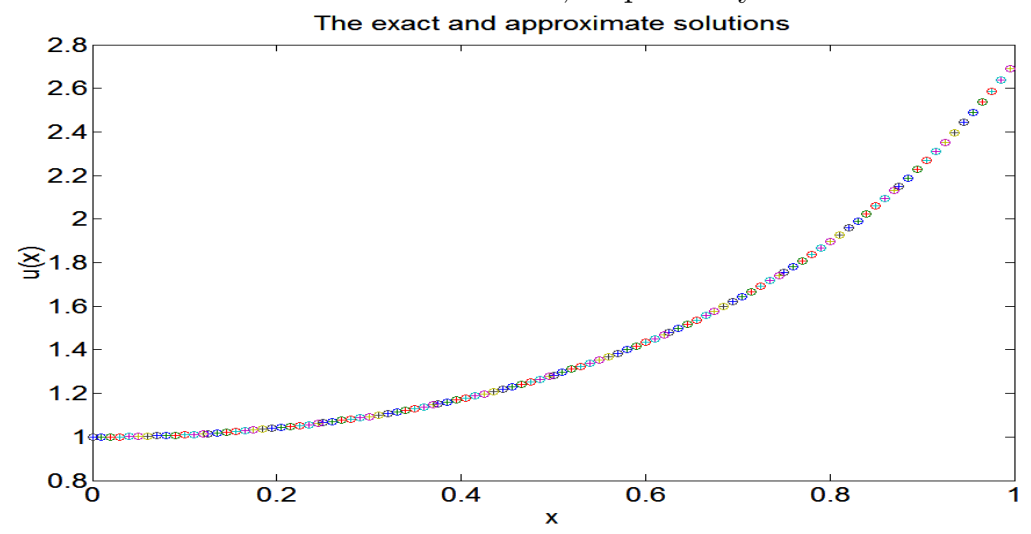

Fig.3. The exact solution and approximate solution with $\mathrm{p}=4, \mathrm{n}=1$ 
Experiment 3: Let $\alpha=2, f(x, u)=u^{5}$ and $g(x)=0$ with $A=1$, Eq.(1.1) can be written as

$$
u^{\prime \prime}+\frac{2}{x} u^{\prime}+u^{5}=0
$$

with initial conditions $u(0)=1, u^{\prime}(0)=0$, which has exact solution $u(x)=\left(1+\frac{x^{2}}{3}\right)^{-1 / 2}$. The nonlinear term $u^{5}$ of Eq.(4.3) is calculated by using the integer power operator (3.18) and the numerical solution of Eq.(4.3) is obtained by using the DLWG method as Table 3 and Fig. 4 described, respectively.

Table 3. Error by using the DLWG method for Example 3

\begin{tabular}{ccc}
\hline$p$ & $n$ & MSE \\
\hline \multirow{2}{*}{3} & 1 & $5.1725 \times 10^{-6}$ \\
& 2 & $1.4354 \times 10^{-7}$ \\
\multirow{2}{*}{4} & 1 & $1.8594 \times 10^{-7}$ \\
& 2 & $2.1757 \times 10^{-9}$ \\
5 & 1 & $1.1337 \times 10^{-7}$ \\
& 2 & $6.1375 \times 10^{-10}$ \\
\hline
\end{tabular}

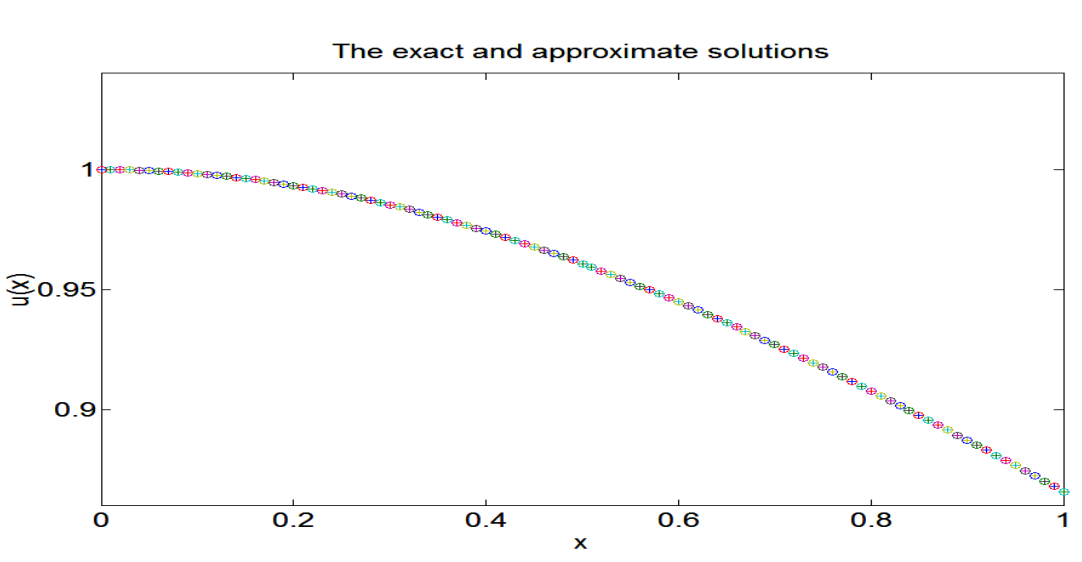

Fig.4. The exact solution and approximate solution with $\mathrm{p}=5, \mathrm{n}=1$

It is noted that Fig. 4 illustrates the exact solution and the numerical approximate solution in space $V_{5,1}$, respectively. Here we only present the Legendre scale coefficients in subspace $V_{3,1}$ as

$$
C_{3,1}=\left[\begin{array}{cccc}
0.6976 & -0.0081 & -0.0020 & 0.0001 \\
0.6479 & -0.015 & -0.0009 & 0.0001
\end{array}\right]
$$

Experiment 4: Solve the nonlinear two-point boundary value problem

$$
u^{\prime \prime}-e^{u}=0,0 \leq x \leq 1,
$$

with boundary conditions $u(0)=u(1)=0$, which is of great interest in hydrodynamics. This question has unique solution [19]

$$
u(x)=-\ln 2+\ln \lambda(x),
$$

where $\lambda(x)=\frac{c}{\cos [0.5 c(x-0.5)]}$ and $c$ is the root of $\left[\frac{c}{\cos (\mathrm{c} / 4)}\right]^{2}=2$.

The nonlinear term $e^{u}$ of Eq.(4.4) is first expanded by Taylor series and then this series are approximated by using the integer power operator in (3.18). Finally, Eq. (4.4) can be converted into systems of nonlinear equations with respect to the scale coefficients as the formulation (3.33). These 
systems are solved by Newton's iterative method and the numerical solution obtained demonstrates the efficiency of the DLWG approach. Furthermore, we use Table 4 and Fig. 5 to illustrate the approximate results. We only present the approximate coefficients in scale space $V_{3,1}$, i.e., $p=3$ and $n=1$, and $c_{3,1 l}$ satisfies

$$
C_{3,1}=\left[\begin{array}{cccc}
-0.2703 & -0.0115 & 0.0031 & -0.0019 \\
-0.2737 & 0.0115 & 0.0031 & 0.0019
\end{array}\right]
$$

Table 4. Error by using the DLWG method for Example 4

\begin{tabular}{cll}
\hline$p$ & $n$ & \multicolumn{1}{c}{ MSE } \\
\hline \multirow{2}{*}{3} & 1 & $2.0737 \times 10^{-3}$ \\
& 2 & $4.3705 \times 10^{-4}$ \\
4 & 2 & $6.1425 \times 10^{-7}$ \\
& 3 & $2.7349 \times 10^{-9}$ \\
5 & 1 & $1.9024 \times 10^{-10}$ \\
& 2 & $8.7131 \times 10^{-11}$ \\
\hline
\end{tabular}

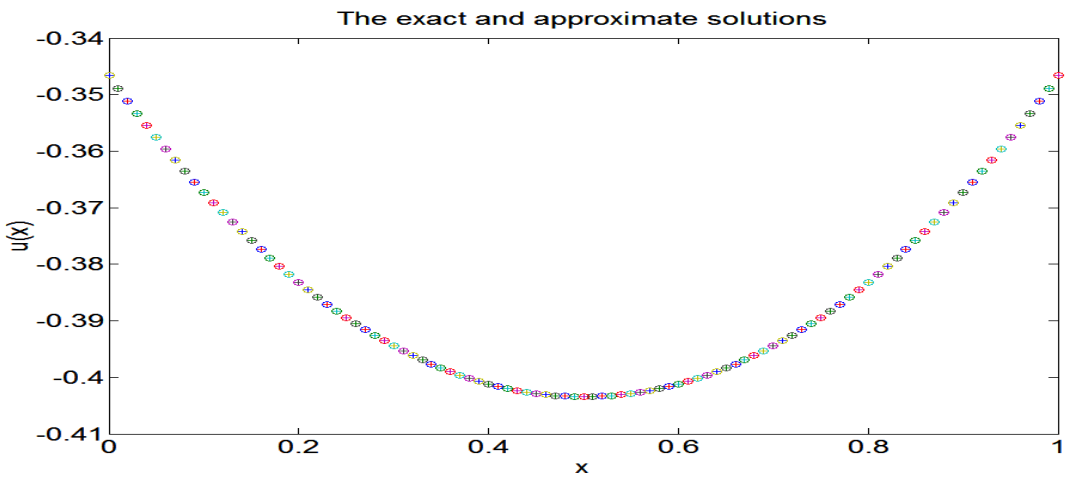

Fig.5. The exact solution and approximate solution with $\mathrm{p}=5, \mathrm{n}=2$

These experiments demonstrate the validity and applicability of the DLWG technique proposed in this article for solving Lane-Emden type equation with less computational complexity. The reason is that we adopt calculations on each element and take advantages of the nice properties of Legendre wavelet bases. Additionally, for high accuracy, the decomposition level $n$ or the degree $p$ of Legendre wavelet functions should be increased.

Remark: Even though we develop the DLWG method to solve Lane-Emden type equation in this article, we take special care that it could be generalized properly to PDEs and integro-differential equations so that we can address these issues in the future.

\section{Conclusion}

In this article, the weak formulation of Eq. (1.1) including the linear and the nonlinear ODEs is derived and a general procedure for calculating each term for the variational formulation (3.3) by using the DLWG approach is clearly presented. Then the scheme is applied to solving the linear and nonlinear 
singular examples. The numerical solutions obtained for these examples show that this approach can effectively solve these problems and are very simple and easy in implementation.

Acknowledgments. This work is funded by Fundamental and Advanced Research Project of Chongqing CSTC of China under grant numbers CSTC2013JCYJA00022 and CSTC2012jjA00018 and supported by Technological Research Program of Chongqing Municipal Education Commission under grant numbers KJ130818 and KJ130810.

\section{References}

1. Davis, H.T. Introduction to Nonlinear Differential and Integral Equations. Dover, New York (1962)

2. Chandrasekhar, S. Introduction to Study of Stellar Structure. Dover, New York (1967)

3. N.T. Shawagfeh, N.T. "Nonperturbative approximate solution for Lane-Emden equation." J. Math. Phys. 34 (9), 4364-4369 (1993)

4. Singh, O.P., Pandey, R.K., Singh, V.K. "An analytic algorithm for Lane-Emden equations arising in Astrophysics using MHAM. Comput." Phys. Commun. 180, 1116-1124 (2009)

5. Wazwaz, A.M. "A new algorithm for solving differential equation Lane-Emden type." Appl. Math. Comput. 118, $287-310(2001)$

6. Liao, S.J. "A new analytic algorithm of Lane-Emden type equations." Appl. Math. Comput. 142, 1-16 (2003)

7. Iqbal, S. and A. Javed, "Application of optimal homotopy asymptotic method for the analytic solution of singular Lane-Emden type equation." Appl. Math. Comput. 217, 7753--7761 (2011)

8. He, J.H. "Variational approach to the Lane-Emden equation." Appl. Math. Comput. 143, 539-541 (2003)

9. Dehghan, M.F., Shakeri, F. "Approximate solution of a differential equation arising in astrophysics using variational iteration method." New Astron. 13, 53-59 (2008)

10. Parand, K., Shahini, M., Dehghan, M. "Rational Legendre pseudospectral approach for solving nonlinear differential equations of Lane-Emden type." J. Comput. Phys. 228, 8830-8840 (2009)

11. Van Gorder, R.A. "An elegant perturbation solution for the Lane-Emden equation of the second kind." New Astron. 16 , 65-67 (2011).

12. Van Gorder, R.A. "Analytical solutions to a quasilinear differential equation related to the Lane-Emden equation of the second kind. Celestial Mech." Dynam. Astron. 109, 137-145 (2011)

13. Razzaghi, M., Yousefi, S. "The Legendre wavelets Operational Matrix of Integration," International Journal of Systems Science. 32, 500-502 (2001)

14. Yousefi, S. "Legendre wavelets method for solving differential equations of Lane-Emden type," Appl. Math. Comput. 181, 1417-1422 (2006)

15. Razzaghi, M., Yousefi, S. "Legendre wavelets method for the solution of nonlinear problems in the calculus of variations." Math. Comput. Model. 34, 45-54 (2001)

16. Mohammadi, F., Hosseini, M.M. "A new Legendre wavelet operational matrix of derivative and its applications in solving the singular ordinary differential equations." Journal of the Franklin Institute. 348, 1787-1796 (2011)

17. Pandey, Rajesh K., Kumar, Narayan. "Abhinav Bhardwaj and Goutam Dutta, Solution of Lane-Emden type equations using Legendre operational matrix of differentiation." Applied Mathematics and Computation. 218, 7629-7637 (2012)

18. Mohammadi, F.M., Hosseini, M.M, Mohyud-din, S.T. "Legendre wavelet Galerkin method for solving ordinary differential equations with nonanalytic solution." Int. J. Syst. Sci. 42 (4), 579-585 (2011)

19. Kumar, S., Sloan, I.H. "A new collection-type method for Hammerstein integral equations." J.Math.Comput. 48, 125-129 (1987)

20. Alpert, B., Beylkin, G., Gines, D., Vozovoi, L. "Adaptive Solution Partial Differential Equations in Multiwavelet Bases." J.Comp.Phys. 182, 149-190 (2002)

21. Wazwaz, A.M. "A new method for solving singular initial value problems in the second-order ordinary differential equations." Appl. Math. Comput. 128, 45-57 (2002)

22. Avudainay agarn, A., Vano, C. "Wavelet-Galerkin method for integro-differential equations." Appl.Numer.Math. $32,247-254$ (2000) 
23. Mohammadi, F., Hosseini, M.M. "Legendre wavelet method for solving linear stiff systems." J. Adv. Res. Differential Equations. 2 (1), 47--57 (2010)

24. Zheng Xiaoyang, Yang Xiaofan, Su Hong, Qiu, Liqiong "Discontinuous Legendre wavelet element method for elliptic partial differential equations." Applied Mathematics and Computation. 218, 3002-3018 (2011)

25. Vidden Chad, Yan, Jue "A new direct discontinuous Galerkin method with symmetric structure for nonlinear diffusion equations." Journal of Computational Mathematics. Vol.31, No.6, 638-662 (2013) 